\title{
ÁREA DE VIDA, DISTRIBUCIÓN POTENCIAL Y ESTADO DE CONSERVACIÓN DE Espostoa frutescens Madsen, 1989 (CACTACEAE)
}

\section{HOME RANGE, POTENTIAL DISTRIBUTION AND CONSERVATION STATUS OF Espostoa frutescens Madsen, 1989 (CACTACEAE)}

\author{
Christian R. Loaiza S. ${ }^{1}$
}

\begin{abstract}
Resumen
Espostoa frutescens, conocido como cactus lanudo del sur del Ecuador, se conoce solo de nueve localidades confirmadas en Ecuador y actualmente se encuentra listado como casi amenazado (NT) por la UICN. Se analiza la distribución, extensión de ocurrencia y el área de vida de esta especie, y se evalúa su estado de conservación actual. Se presenta el primer modelo de hábitat / nicho basado en los registros conocidos y el primer acercamiento geográfico para esta especie. En base a los nueve registros confirmados se construyó un modelo de hábitat / nicho de máxima entropía determinando previamente las variables ambientales de mayor importancia para el modelo. Se obtuvo un índice predictivo aceptable (0.971) para el modelo final. Se determinó las zonas de mayor probabilidad de ocurrencia para esta especie y se estimó el área de vida mediante el método del polígono mínimo convexo (PMC). El área prioritaria más importante identificada para la conservación de esta especie se sitúa al sur del Ecuador, entre las provincias de Azuay y Loja. Se recomienda utilizar el presente modelo como una herramienta útil para futuras evaluaciones de la conservación de esta especie.

Palabras clave: Ecuador, Espostoa frutescens, modelo de nicho ecológico, área de vida.
\end{abstract}

\begin{abstract}
Espostoa frutescens, known as Ecuadorian southern woolly cactus, is known only from nine confirmed localities in the south of Ecuador and is currently listed as near threatened (NT) by the IUCN. We analyze the distribution, occurrence extension and home range of this species, and evaluate its current conservation status. Based on the nine known records and the first geographic approach to this species, a habitat / niche model is presented, which was built by previously determining the most important environmental variables for the model. An acceptable predictive index (0.971) was obtained for the final model. The areas of major probability of occurrence for this species were determined and the home range was estimated by the use of the polygon minimum convex method (PMC). The most important priority area identified for the conservation of this species is located in the south of Ecuador, between the provinces of Azuay and Loja. We recommend the use of the present model as a useful tool for future evaluations of conservation of this species.
\end{abstract}

Key words: Ecuador, Espostoa frutescens, ecological niche model, home range.

\section{Introducción.}

La familia Cactaceae es endémica de América (Anderson, 2001). Su distribución comprende todo el continente, desde el nivel del mar hasta las zonas altoandinas (Nyffeler, 2002; Arakaki, 2011). En Ecuador, esta familia se encuentra representada por 16 géneros y 43 especies nativas (Loaiza et al., 2009), de las cuales 13 especies son endémicas para el país, sin incluir las Islas Galápagos (Madsen, 1989, 2002).

El género Espostoa Britton y Rose (1920), se encuentra distribuido en gran parte de la región centro - norte andina, desde el sur de Ecuador hasta Bolivia (Britton \& Rose, 1920; Madsen, 1989; Ostolaza, 2014). Según la clasificación de Hunt (2006), este género se encuentra representado por 11 especies y seis subespecies. A nivel de Perú, el género está representado por 10 especies y cinco subespecies (Ostolaza, 2014). En Ecuador, la presencia de este género se reduce a dos especies y una subespecie, reconociéndose tan solo un endemismo en la región sur del país (Loaiza et al., 2009; Madsen, 1989, 2002; Madsen \& Aguirre, 2004).

Este género se encuentra representado por plantas arbustivas o arbóreas, entre 0.6 - $7 \mathrm{~m}$ de altura, muy ramificadas. El tronco puede estar presente o ausente. Los segmentos / ramas del tallo pueden formar candelabros de 0.4 - $4 \mathrm{~m}$ de largo, cubiertos de lana blanca. El número de costillas suele variar entre 18 - 


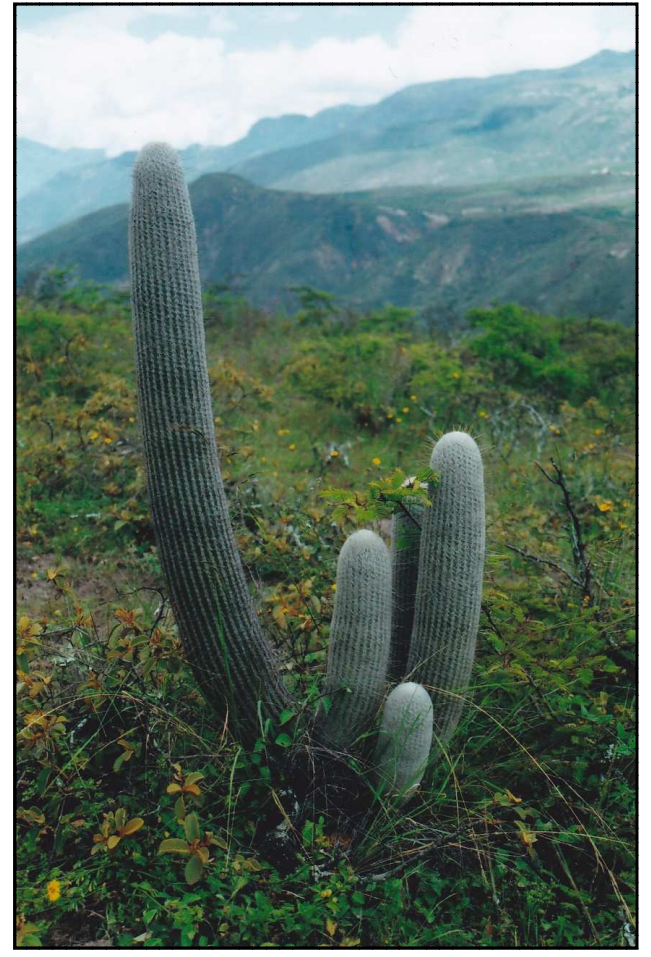

Figura 1. Espostoa frutescens.

28, según la especie. Las espinas son numerosas y cortas. Las partes florales de la planta se encuentran agrupadas y protegidas en zonas laterales (cefalios) de los segmentos del tallo, compuestas por pelos lanosos de $2-3 \mathrm{~cm}$ de largo. Las flores pueden ser de color blanco o púrpura, de $4-6 \mathrm{~cm}$ de largo, actinomorfas, con brácteas pequeñas y tricomas, de hábito nocturno. Los frutos son redondos, de color verde, blanco o rojo, entre $1-3 \mathrm{~cm}$ de ancho. Las semillas pueden ser ovoides o obovoides, verrugosas o lisas, de aspecto opaco o brillante, numerosas y de coloración negra (Britton \& Rose, 1920; Madsen, 1989, 2002; Ostolaza, 2014).

Espostoa frutescens se caracteriza por ser una planta arbórea de tamaño mediano, profusamente ramificada desde la base, de 1.5 - $2 \mathrm{~m}$ de alto (Figura 1). El tronco es ausente, su coloración es verde oscura. El número de costillas varía entre 22 - 28. Las areolas son de forma elíptica ( 45 - 50 por costilla), de 3 - 4 $\mathrm{mm}$ de largo. Espina central solitaria, de $1-2.5 \mathrm{~cm}$ de largo x $0.5-0.75 \mathrm{~mm}$ de ancho. Las espinas radiales son de color amarillo pálido, aciculares y difusas (38 40 por areola). Las flores son blancas, en forma de embudo, de $4-4.5 \mathrm{~cm}$ de largo x $3.5-4 \mathrm{~cm}$ de ancho. Los frutos son de color verde, su forma es globosa (baya), de $16-20 \mathrm{~mm}$ de largo x $15-25 \mathrm{~mm}$ de ancho. El período de floración y fructificación de esta especie ocurre entre los meses de octubre, noviembre y diciembre. Esta especie se caracteriza por tener un síndrome de quiropterofilia bastante estricto. Sus flores son nocturnas y suelen ser visitadas por murciélagos de los géneros Anoura y Glossophaga. Las semillas son pequeñas, de forma obovoide, verrugosas y de coloración negra. Su número es abundante (Madsen, 1989, 2002).

El tipo de formaciones vegetales en las cuales se distribuye esta especie corresponden a Bosque de neblina montano de los andes occidentales, Bosque semideciduo piemontano de la costa y Matorral seco montano de los Andes del sur (Valencia et al., 1999). Su rango altitudinal se sitúa entre los $800-2600 \mathrm{~m}$ de altitud (Herbarios: AAU, HUTPL, LOJA, QCA, QCNE y UDA). Esta especie se encuentra situada dentro del trazo generalizado (distrito) denominado como "Trazo interandino del sur" (Loaiza \& Morrone, 2011), el cual se ubica entre las provincias de Loja, Azuay y parte de la provincia del Cañar. Su área de vida corresponde principalmente a valles secos y zonas de matorral seco espinoso (Aguirre et al., 2006; Loaiza, 2013; Loaiza et al., 2009; Madsen, 2002). Esta especie suele formar asociaciones con otras especies de cactus como Armatocereus sp. y Opuntia pestifer. Al parecer esta especie conforma un grupo de cactáceas endémicas exclusivas de las cuencas del río Jubones y del río León en el suroccidente del Ecuador, entre las que destacan Armatocereus sp. Cleistocactus leonensis, Weberocereus rosei, entre otras.

Esta especie se encuentra poco representada en las colecciones de herbarios del Ecuador, razón por la cual ha resultado un poco difícil el poder determinar su correcta distribución en algunas evaluaciones anteriores, así como también evaluar su estado de conservación. Colecciones adicionales de esta especie,

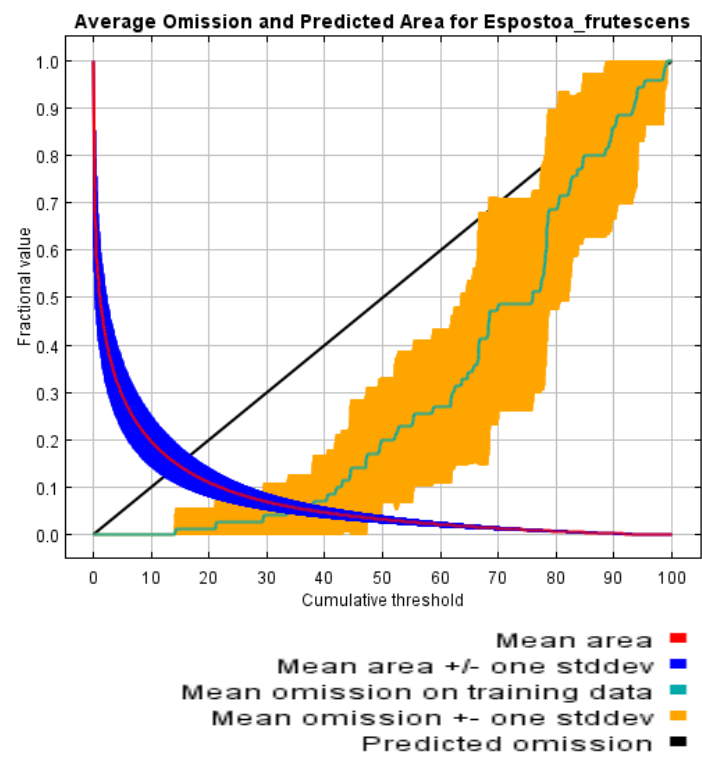

Figura 2. Curva de omisión / predicción para Espostoa frutescens. 
provenientes de las mismas localidades en Ecuador se encuentran depositadas también en los herbarios MO y ZSS.

El objetivo principal del presente trabajo es presentar una evaluación actualizada del estado de conservación y la distribución de Espostoa frutescens en su área de distribución dentro del Ecuador.

\section{Materiales y métodos.}

Trabajo de campo.

Las primeras evaluaciones del estado de conservación de esta especie se dieron en base a las observaciones realizadas por Jens E. Madsen, recogidas en las dos ediciones del Libro Rojo de Plantas Endémicas del Ecuador. De acuerdo a la última evaluación realizada en el 2011, esta especie se consideró bajo la categoría En Peligro (EN) de acuerdo al criterio B1 ab (iii) de la UICN (Madsen \& Montúfar, 2011). En aquel tiempo, las únicas colecciones conocidas sobre esta especie provenían de cuatro localidades situadas entre las provincias de Azuay y Loja (J. E. Madsen et al. / 61064, 61085, 61135 y 7416 / AAU, QCA, QCNE y LOJA). Todas las colecciones señaladas fueron realizadas durante el período 1985 - 2000. Posterior a estos primeros registros, se realizaron nuevas colecciones a partir del año 2011 y se analizó detenidamente el estado poblacional y la distribución de esta especie en base a nuevas localidades. El nuevo material botánico obtenido fue depositado en el herbario de la Universidad del Azuay (UDA) y en el herbario de la Universidad Técnica Particular de Loja (HUTPL). Las nuevas colecciones depositadas en el herbario HUTPL fueron realizadas por el autor del presente trabajo: (CL - 018, 024 y 025). Finalmente, durante el mismo año 2011, se realizó un taller para la evaluación del estado de conservación de las cactáceas de la región andina

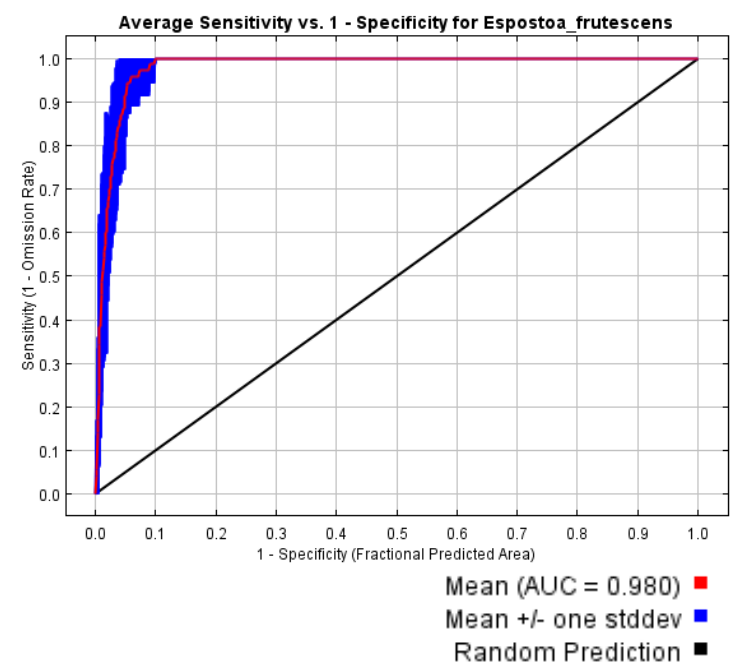

Figura 3. Curva de sensibilidad / especiticidad para Espostoa frutescens.

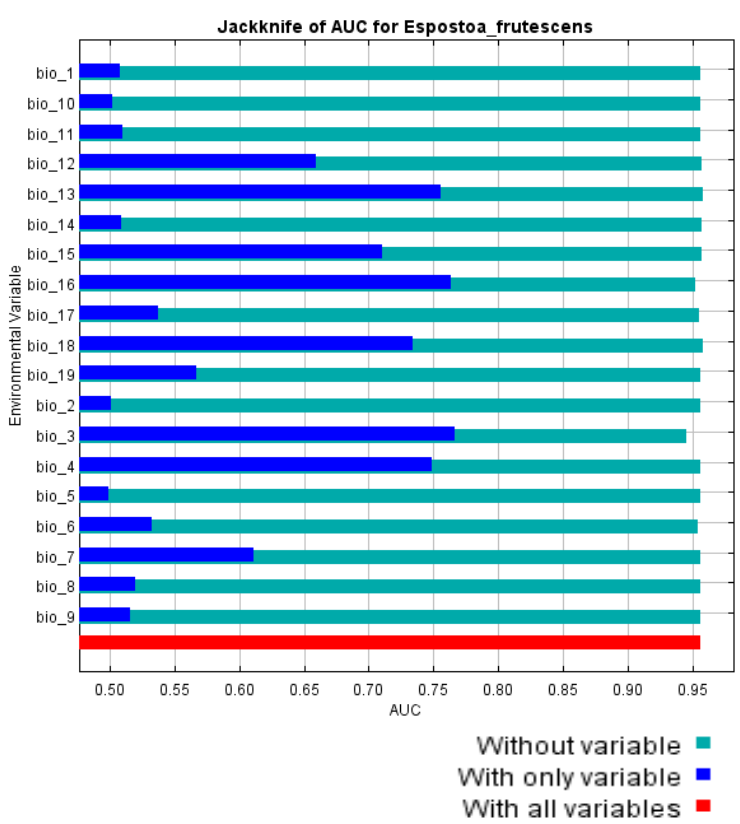

Figura 4. Test de jackknife para Espostoa frutescens.

en el marco del proyecto "Global Cactus Assesment", financiado por la Universidad de Sheffield y por la IUCN, cuyos resultados fueron publicados en el año 2015 (Goettsch et al., 2015).

Modelamiento distributivo.

Para analizar la distribución potencial de esta especie se utilizó el programa Maxent, debido principalmente a su capacidad de poder combinar su aplicación con el uso de herramientas SIG (Arcgis) para poder generar mapas de distribución actual y potencial de especies nativas, lo cual resulta fundamental para poder evaluar el estado de conservación de las especies y para evaluar sitios potenciales para el desarrollo de iniciativas de conservación y restauración (Morales, 2012). Este programa estima la probabilidad de ocurrencia de una especie buscando la distribución de máxima entropía (lo más uniforme posible), a partir de datos de presencia y variables ambientales representadas en un mapa raster, todo ello sujeto a la condición de que el valor esperado de cada variable ambiental, según la distribución obtenida, coincida con su media empírica (Merow et al., 2013; Morales, 2012; Phillips \& Dudik, 2008; Riquelme, 2013).

Como paso previo para el modelamiento se procedió a descargar un conjunto de variables ambientales del world clim (www.worldclim.org), las cuales son de amplia cobertura global y comprenden diferentes tipos de factores bioclimáticos (temperatura, precipitación y altitud) en formato raster, el cual permite su uso en Maxent. Se descargó el archivo bioclima 30 s en formato ESRI grid $(\sim 1$ $\mathrm{km})$ y se realizó un recorte de las variables para las 
Enero - Julio 2017

provincias de Azuay, Loja y El Oro (Tabla 1), con el fin de contar con un juego de variables ambientales específicamente diseñado para la región sur del Ecuador.

Para obtener la distribución potencial de esta especie se construyó una base de datos en Excel, la cual contó con nueve registros obtenidos en base a información personal / trabajo de campo, complementada con los registros de los principales herbarios del Ecuador y algunas bases de datos de herbarios internacionales (Tabla 2). Esta información posteriormente fue debidamente georreferenciada y transformada a un archivo de texto CSV (delimitado por comas). Debido al reducido número de registros disponibles, se siguió la metodología aplicada por Giménez et al., 2015, la cual consiste en desarrollar 10 réplicas para el modelo, con el $25 \%$ de los registros usados como test data, 500 iteraciones y 10000 puntos de respaldo "background points" con la finalidad de obtener una mayor precisión en el modelamiento. Esta metodología se aplicó para hacer un "jackknife" y determinar las variables ambientales que mejor contribuyen con el modelamiento (Figuras 2 y 3 ). Luego de registrar las variables ambientales con menor valor de contribución para el modelamiento (Figura 4), se volvió a correr nuevamente el modelamiento y se seleccionó como archivo de salida "output" un modelo logístico en formato "asc", ya que este modelo es el más fácil de conceptualizar y permite obtener como resultado un mapa binario, con valores entre 0 y 1 , en donde 1 es el valor que permite visualizar el área de distribución potencial / modelo predictivo de una especie.

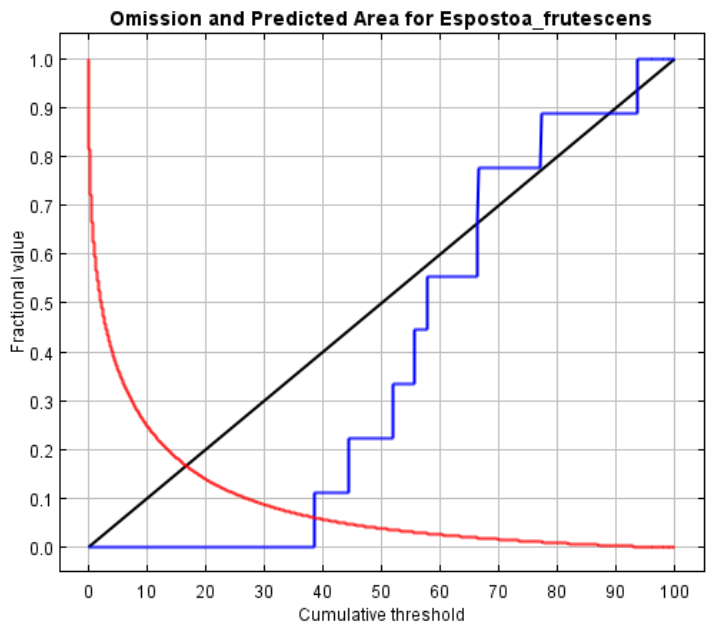

Fraction of background predicted Omission on training samples Predicted omission

Figura 5. Modelo de curva acumulativa (AUC) para Espostoa frutescens.

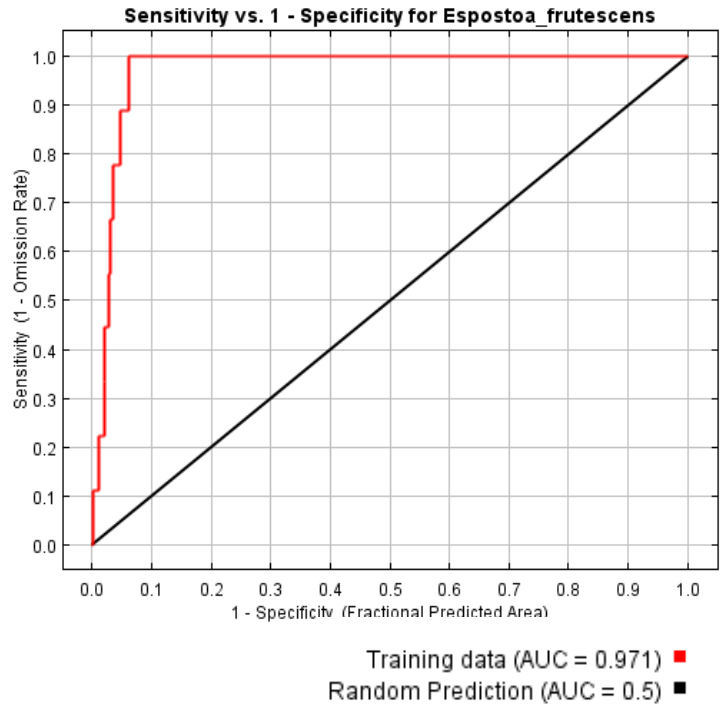

Figura 6. Valor de AUC obtenido para Espostoa frutescens.

El propósito de la gráfica de la curva operacional (Curva ROC) obtenida para el modelamiento es señalar, en el caso de la curva roja (entrenamiento), el ajuste del modelo a los datos del muestreo y, en el caso de la curva azul (test), el grado de ajuste del modelo a los datos y supone el test real del poder predictivo del modelo. La línea negra representa a su vez, la línea esperada si el modelo no fuese mejor que "por azar". El valor del AUC representado en la curva ROC va de 0 a 1 y se considera que mientras más cerca se encuentre el valor obtenido a 1 , mejor es el criterio de aceptación del modelamiento obtenido.

Como análisis estadístico, se evaluaron los test dependiente e independiente, usando para ellos como umbral de corte los valores de P en 1, 5 y 10 bajo el área de curva AUC (Tabla 3). Para el umbral de corte, se seleccionó la opción para el mínimo de presencia de los puntos de entrenamiento "Minimum training presence", lo cual permite obtener modelos bien precisos y además, permite generar como resultado archivos en formato "shapefile" que permiten realizar mapas distributivos.

Área de vida.

Para estimar el área de vida se utilizó una herramienta disponible en ArcGis llamada Xtools Pro, la cual permite crear polígonos en base a los registros de una especie y además, estimar su área de vida en $\mathrm{km}^{2}$.

Los mapas finales fueron realizados con el programa ArcGis 10.3.

\section{Resultados.}

Distribución y biogeografía.

El modelamiento realizado en Maxent para esta especie tuvo un nivel de aceptación bastante óptimo 


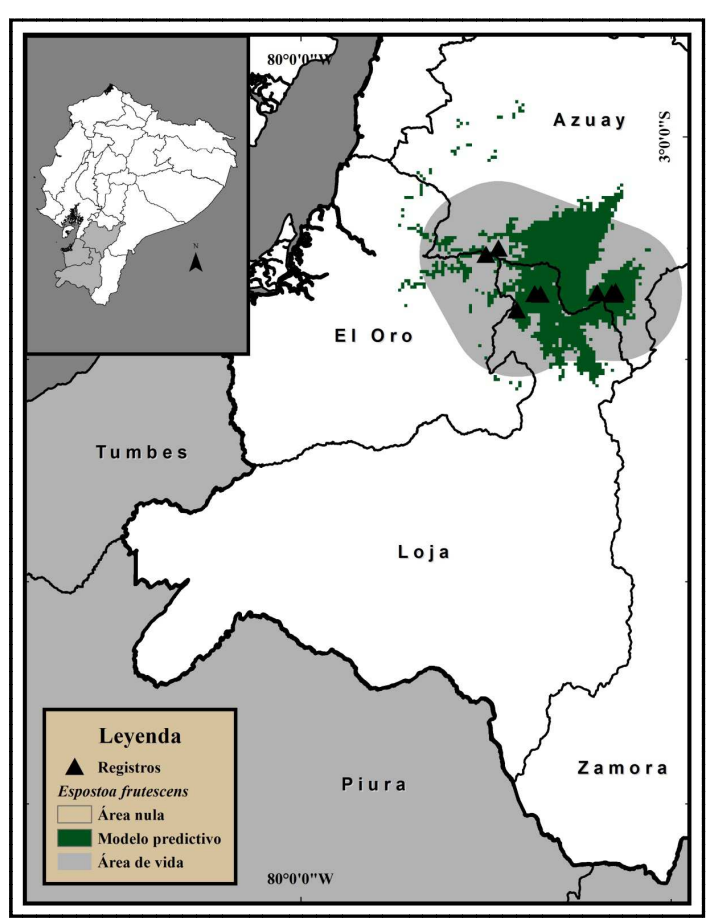

Figura 7. Área de vida (PMC) y distribución potencial de Espostoa frutescens.

(Figura 5). El valor de AUC obtenido fue de $0.971 \mathrm{y}$ el grado de predicción al azar fue de 0.5 , lo cual indica un nivel de confianza bastante aceptable (Figura 6).

De las variables utilizadas, cuatro variables con valores superiores al $1 \%$ contribuyeron con el modelamiento, lo cual representa el $99.2 \%$ de la varianza explicada (Tabla 1). Las variables ambientales con mayor influencia para la distribución de E. frutescens fueron: bio_3 (50.5\%) y bio_16 (42.9 $\%)$ (Tabla 1).

La distribución de esta especie ocurre entre las provincias de Azuay, Loja y El Oro, sin embargo, en base al modelamiento obtenido (Figura 7) se establece que las áreas de mayor ocurrencia para esta especie se sitúan principalmente en los cantones de Santa Isabel y Oña (Azuay) y Saraguro (Loja). Dentro de la provincia de El Oro, esta especie es particularmente abundante en la cuenca del río Jubones. Su distribución se extiende de forma prolongada a lo largo del valle interandino de Yunguilla (Santa Isabel) en la provincia del Azuay hasta llegar al cantón Pasaje en la provincia del Oro, en donde es una especie bastante común.

\section{Discusión.}

Estado de conservación.

En base a las observaciones realizadas durante el 2011, se concluyó que el estado poblacional de esta especie se ha mantenido estable en varias localidades dentro de su área de distribución durante los últimos años, siendo bastante común en algunas localidades, principalmente en el valle de Yunguilla (Santa Isabel) y a lo largo de la vía Santa Isabel - Pasaje. No se evidenció en ningún momento que esta especie sea aprovechada de alguna forma ya sea como planta ornamental o por algún otro medio y tampoco se detectaron amenazas potenciales para la conservación de sus poblaciones, ni se evidenció que existan poblaciones severamente fragmentadas.

El área de vida establecida para esta especie se estimó en un valor aproximado de $3383.7 \mathrm{~km}^{2}$, el cual fue obtenido en base a un polígono creado con la ayuda del programa Xtools Pro (Figura 7), utilizando todos los registros conocidos. Sin embargo, al confrontar el polígono creado con el modelamiento obtenido en Maxent, se considera que el área de vida de esta especie es menor al valor obtenido y se podría estimarlo en 2500 - $2800 \mathrm{~km}^{2}$. En base a ello, de acuerdo al criterio B1 ab (iii) de la UICN, esta especie debería permanecer dentro de la categoría de especies consideradas en peligro (EN), sin embargo, dado que las poblaciones de esta especie no se encuentran severamente fragmentadas y el número de registros es superior a cinco, se considera que esta especie debe estar asignada en la categoría de especies poco amenazadas "Near Threatened" (NT), además de que su área de vida bien podría superar o al menos llegar a los $3500 \mathrm{~km}^{2}$ si se consideran zonas de probable distribución en la provincia de El Oro.

A pesar de la ausencia de amenazas potenciales para la conservación de esta especie, se debe señalar que esta especie no se encuentra presente dentro de ninguna área protegida y no existe ningún organismo en el Ecuador que se encargue de monitorear el estado poblacional de las distintas especies de cactáceas en el país. Por ello se recomienda y a la vez se sugiere realizar monitoreos permanentes a fin detectar cualquier tipo de alteración o amenaza que pueda afectar las poblaciones de esta especie y en caso de tener alguna evidencia, tomar las medidas del caso a fin de poder asegurar su supervivencia a largo plazo, más aun considerando que se trata de una especie endémica para el Ecuador. También se sugiere buscar la implementación de algún área protegida o bosque protector que permita la conservación de las poblaciones más importantes de esta especie dentro de su área de distribución. Por último, es necesario desarrollar los estudios necesarios para conocer al detalle su biología reproductiva e interacción con especies polinizadoras.

\section{Agradecimientos.}

El autor de este trabajo desea expresar su principal agradecimiento a Bárbara Goettsch por las observaciones y valiosos comentarios realizados al presente artículo. Dos revisores anónimos realizaron observaciones y sugerencias al manuscrito inicial. Al personal del herbario de la Universidad Técnica 
Particular de Loja (HUTPL) por su soporte y apoyo durante las distintas salidas de campo realizadas en la provincia de Loja. Un agradecimiento al personal del herbario LOJA, QCA y QCNE por permitirme revisar las distintas colecciones de cactáceas depositadas en sus instalaciones y por las facilidades brindadas.

\section{Literatura citada.}

Aguirre Z., Kvist L.P. \& Sánchez O. 2006. Bosques secos en Ecuador y su diversidad. Páginas 162-187. En: M. Moraes, B. Øllgaard, L. P. Kvist, F. Borchsenius y H. Balslev (eds.). Botánica Económica de los Andes Centrales. Universidad Mayor de San Andrés, La Paz.

Anderson E.W. 2001. The Cactus Family. O. R. Timber Press, Portland. 776 pp.

Arakaki M. 2011. Contemporaneus and recent radiations of the world's major succulent plant lineages. Proceedings of the National Academy of Sciences. 108(20): 83798384.

Britton N.L. \& Rose J.N. 1920. The Cactaceae: Descriptions and illustrations of plants in the cactus family. Vol. 2 . The Carnegie Institution of Washington. Washington D. C. $241 \mathrm{pp}$.

Giménez A.L., Giannini N.P., Schiaffini M.I. y Martin G.M. 2015. Geographic and potential distribution of a poorly known South American bat, Histiotus macrotus (Chiroptera: Vespertilionidae). Acta Chiropterologica 17 (1): 143 - 158.

Goettsch B., Hilton-Taylor C., Cruz-Piñón G., Duffy J.P., Frances A., Hernández H.M., Inger R., Pollock C., Schipper J., Superina M., Taylor N.P., Tognelli M., Abba A.M., Arias S., Arreola-Nava H.J., Baker M.A., Bárcenas R.T., Barrios D., Braun P., Butterworth C.A., Búrquez A., Cáceres F., Chazaro-Basañez M., CorralDíaz R., Perea M., Demaio P.H., Duarte de Barros W.A., Durán R., Yancas L.F., Felger R.S., Fitz-Maurice B., Fitz-Maurice W.A., Gann G., Gómez-Hinostrosa C., González-Torres L.R., Griffith M.P., Guerrero P.C., Hammel B., Heil K.D., Hernández-Oria J.G., Hoffmann M., Ishihara M.I., Kiesling R., Larocca J., León-De la Luz J.L., Loaiza Salazar C.R., Lowry M., Machado M.C., Majure L.C., Martínez J.G., Martorell C., Maschinski J., Méndez E., Mittermeier R.A., Nassar J.M., Negrón-Ortiz V., Oakley L.J., Ortega-Baes P., Pin A.B., Pinkava D.J., Porter J.M., Puente-Martinez R., Roque-Gamarra J., Saldivia P., Sánchez E., Smith M., Sotomayor J.M., Stuart S.N., Tapia J.L., Terrazas T., Terry M., Trevisson M., Valverde T., Van Devender T.R., Véliz-Pérez E., Walter H.E., Wyatt S.A., Zappi D., Zavala-Hurtado J.A. \& Gastón K.J. 2015. High proportion of cactus species threatened with extinction. Nature Plants. 15142: 1-7.

Hunt D., Taylor N. \& Charles G. 2006. The New Cactus Lexicon. DH Books. U.K.
Loaiza C. 2013. Espostoa frutescens. The IUCN Red List of Threatened Species 2013: http://dx.doi.org/10.2305/IUCN.UK.20131.RLTS.T151960A581156.en Downloaded on 02 July 2016.

Loaiza C.R., Aguirre Z.H. \& Jadán O. 2009. Estado del conocimiento actual de la familia Cactaceae en el Ecuador. Boletín de la Sociedad Latinoamericana y del Caribe de Cactáceas y otras Suculentas. 6(3): 11-22.

Loaiza C.R. \& Morrone J.J. 2011. Análisis panbiogeográfico de algunas Cactaceae del Ecuador. Gayana Botánica. 68(1): 219-224.

Madsen J.E. 1989. Cactaceae. En G. Harling y L. Anderson (eds.), Flora of Ecuador 35: 1-79.

Madsen J.E. 2002. Cactus en el sur del Ecuador. Páginas 289-303. En: Z. Aguirre M., J.E. Madsen, E. Cotton y H. Balslev (eds.). Botánica Austroecuatoriana: Estudios sobre los Recursos Vegetales en las Provincias de El Oro, Loja y Zamora Chinchipe. Ediciones Abya - Yala. Quito.

Madsen J.E. \& Aguirre Z.H. 2004. Cactus novelties from southern Ecuador. Nordic Journal of Botany. 23(1): 2129.

Madsen J.E. \& Montúfar R. 2011. Cactaceae. Páginas 249252. En: S. León - Yánez, R. Valencia, N. Pitman, L. Endara, C. Ulloa y H. Navarrete (Eds.). 2011. Libro Rojo de las Plantas Endémicas del Ecuador. Segunda Edición. Publicaciones del Herbario QCA, Pontificia Universidad Católica del Ecuador, Quito.

Merow C., Smith M.J. \& Silander J.A. 2013. A practical guide to Maxent for modeling species' distributions: what it does, and why inputs and settings matter. Ecography. 36: 1058-1069.

Morales N. 2012. Modelos de distribución de especies: Software Maxent y sus aplicaciones en conservación. Revista Conservación Ambiental. 2(1): 1-3.

Nyffeler R. 2002. Phylogenetic relationships in the cactus family (Cactaceae) based on evidence from TRNK/MATK and TRNL/TRNF sequences. American Journal of Botany. 89(2): 312-326.

Ostolaza C. 2014. Todos los cactus del Perú. Lima, Perú. $538 \mathrm{Pp}$.

Phillips S.J., Anderson R.P. \& Schapire R.E. 2006. Maximum entropy modeling of species geographic distributions. Ecological Modeling. 190: 231-259.

Phillips S.J. \& Dudík M. 2008. Modeling of species distributions with Maxent: new extensions and a comprehensive evaluation. Ecography. 31: 161-175.

Riquelme J.D. 2013. Técnicas para el análisis de patrones de uso del espacio en felinos americanos (Mammalia: Carnivora). Gestión Ambiental. 25: 53-84.

Valencia R.N., Cerón C., Palacios W. \& Sierra R. 1999. Las formaciones naturales de la Sierra del Ecuador. Páginas 79-108. En: R. Sierra (ed.). Propuesta preliminar de un sistema de clasificación vegetal para el Ecuador Continental. Proyecto INEFAN / GEF - BIRF y EcoCiencia, Quito. 
Tabla 1: Porcentaje de contribución (\% C) e importancia de la permutación (PI) de cada variable para el modelamiento logístico y potencial.

\begin{tabular}{llcc}
\hline \multirow{2}{*}{ Variables } & \multicolumn{1}{c}{ Descripción } & \multicolumn{2}{c}{ Modelamiento } \\
\cline { 3 - 4 } & & \% C & PI \\
\hline bio_1 & Temperatura media anual & - & - \\
bio_2 & Oscilación diurna de la temperatura & - & - \\
bio_3 & Isotermalidad & 50.5 & 46.9 \\
bio_4 & Estacionalidad de la temperatura (\%) & 0 & 0 \\
bio_5 & Temperatura máxima del período más cálido & - & - \\
bio_6 & Temperatura mínima del período más frío & 1.3 & 7.7 \\
bio_7 & Oscilación anual de la temperatura & 0.8 & 0 \\
bio_8 & Temperatura media del cuatrimestre más lluvioso & 0 & 0 \\
bio_9 & Temperatura media del cuatrimestre más seco & 4.5 & 0 \\
bio_10 & Temperatura media del cuatrimestre más cálido & - & - \\
bio_11 & Temperatura media del cuatrimestre más frío & - & - \\
bio_12 & Precipitación anual (mm) & 0 & 0 \\
bio_13 & Precipitación del período más lluvioso & 0 & 0 \\
bio_14 & Precipitación del período más seco & - & - \\
bio_15 & Estacionalidad de la precipitación (\%) & 0 & 0 \\
bio_16 & Precipitación del cuatrimestre más lluvioso & 42.9 & 45.4 \\
bio_17 & Precipitación del cuatrimestre más seco & 0 & 0 \\
bio_18 & Precipitación del cuatrimestre más cálido & 0 & 0 \\
bio_19 & Precipitación del cuatrimestre más frío & 0 & 0 \\
Total & & 100 & - \\
\hline
\end{tabular}

Tabla 2: Registros confirmados de Espostoa frutescens utilizados en el modelamiento potencial del hábitat.

\begin{tabular}{|c|c|c|c|c|}
\hline Localidad & Latitud & Longitud & $\begin{array}{r}\text { Altitud } \\
(\mathrm{m})\end{array}$ & Referencia \\
\hline Vía Santa Isabel - Pasaje (Azuay) & $03^{\circ} 18^{\prime}$ & $79^{\circ} 28^{\prime}$ & 1018 & Herbario AAU \\
\hline Vía Cuenca - Loja (Azuay) & $03^{\circ} 25^{\prime}$ & $79^{\circ} 09^{\prime}$ & 1965 & Herbario AAU \\
\hline No determinada (El Oro) & $03^{\circ} 19^{\prime}$ & $79^{\circ} 30^{\prime}$ & 898 & Herbario AAU \\
\hline Vía Cuenca - Oña (Azuay) & $03^{\circ} 25^{\prime} 12.0^{\prime \prime}$ & $79^{\circ} 12^{\prime} 00.0^{\prime \prime}$ & 2492 & Herbario AAU \\
\hline Vía Udushi - Santa Isabel (Loja) & $03^{\circ} 28^{\prime}$ & $79^{\circ} 25^{\prime}$ & 2530 & Herbario Loja \\
\hline Km. 76 de la vía Oña - Cuenca (Azuay) & $03^{\circ} 25^{\prime} 27.3^{\prime \prime}$ & $79^{\circ} 09^{\prime} 07.6^{\prime \prime}$ & 2124 & Herbario HUTPL \\
\hline Vía Manú - Udushi (Loja) & $03^{\circ} 25^{\prime} 22.2^{\prime \prime}$ & $79^{\circ} 22^{\prime} 06.0 ”$ & 1754 & Herbario HUTPL \\
\hline Vía Manú - Udushi (Loja) & $03^{\circ} 25^{\prime} 26.0^{\prime \prime}$ & $79^{\circ} 21^{\prime} 07.7^{\prime \prime}$ & 1420 & Herbario HUTPL \\
\hline Vía Oña - Susudel & $03^{\circ} 25^{\prime} 13.0^{\prime \prime}$ & $79^{\circ} 09^{\prime} 37.0^{\prime \prime}$ & 1854 & Herbario UDA \\
\hline
\end{tabular}

Tabla 3: Resultados de las áreas predictivas para los valores de corte acumulativo 1, 5, 10 y valor de corte mínimo generados para el modelamiento.

\begin{tabular}{cc}
\hline Valores de corte & Modelamiento predictivo \\
\cline { 2 - 2 } acumulativo & Fraccional de área predictiva \\
\hline 1 & 0.605 \\
5 & 0.365 \\
10 & 0.249 \\
Mínimo & 0.061 \\
\hline
\end{tabular}

\footnotetext{
${ }^{1}$ Instituto de Ciencias Biológicas “Antonio Raimondi”, Facultad de Ciencias Biológicas, Universidad Nacional Mayor de San Marcos, Lima, Perú / Representante regional de la Sociedad Latinoamericana de Cactáceas y Suculentas en el Ecuador. christian.loaiza@unmsm.edu.pe.
} 